\title{
Hsa_circ_NOTCH3 regulates ZNF146 through sponge adsorption of miR-875-5p to promote tumorigenesis of hepatocellular carcinoma
}

\author{
Lei Bao ${ }^{1 \#}$, Min Wang ${ }^{2 \#}$, Qiqi Fan ${ }^{3}$ \\ ${ }^{1}$ Department of Pathology, The Affiliated Yantai Yuhuangding Hospital of Qingdao University, Yantai, China; ${ }^{2}$ Department of Pathology, Qishan \\ (Infectious Disease) Hospital of Yantai, Yantai, China; ${ }^{3}$ Department of Liver Disease, Qingdao No. 6 People's Hospital, Qingdao, China \\ Contributions: (I) Conception and design: All authors; (II) Administrative support: All authors; (III) Provision of study materials or patients: All \\ authors; (IV) Collection and assembly of data: All authors; (V) Data analysis and interpretation: All authors; (VI) Manuscript writing: All authors; (VII) \\ Final approval of manuscript: All authors. \\ \#These authors contributed equally to this work. \\ Correspondence to: Qiqi Fan. Department of Liver Disease, Qingdao No. 6 Peoples' Hospital, 9 Fushun Road, Qingdao 266033, China. \\ Email: qug2045@163.com.
}

Background: To explore the specific mechanism of circular RNA (circRNA) in the occurrence and development of hepatocellular carcinoma (HCC), and provide new ideas for its diagnosis and treatment.

Methods: Reverse transcription-quantitative polymerase chain reaction (RT-qPCR) was for evaluating the expression of circ_NOTCH3 in liver cancer tissues and matched normal tissues and related cell lines. After overexpression or co-expression of circ_NOTCH3 or microRNA (miRNA) in cells, the changes in cell function were analyzed. Bioinformatics analysis and dual luciferase report analysis were utilized to predict and verify the binding site between circ_NOTCH3 and miRNA. Western blotting was applied to detect gene expression alterations. Additionally, in vivo tumor growth was also utilized to further assess the influence of knocking-down circ_NOTCH3 on the progression of HCC.

Results: It was confirmed circ_NOTCH3 was highly expressed in HCC specimens and cells. The proliferation, migration, invasion, and oxaliplatin-resistance potential of HCC could be restrained by silencing circ_NOTCH3 or by ectopic expression of miR-875-5p in vitro. In terms of mechanism, circ_ NOTCH3 directly binds to miR-875-5p, regulating its activity by targeting the 3'-UTR of ZNF146. Overexpression of circ_NOTCH3 evidently overturned the diminishing influence of miR-875-5p mimics on HCC cells.

Conclusions: As an oncogene, circ_NOTCH3 can trigger the proliferation, invasion, migration, and oxaliplatin resistance of HCC cells through the miR-875-5p/ZNF146 axis, and may be a promising target for the treatment of HCC.

Keywords: Hepatocellular carcinoma (HCC); circ_NOTCH3; miR-875-5p; ZNF146

Submitted Aug 05, 2021. Accepted for publication Oct 19, 2021.

doi: 10.21037/jgo-21-567

View this article at: https://dx.doi.org/10.21037/jgo-21-567

\section{Introduction}

Hepatocellular carcinoma (HCC) is the most common malignant liver cancer and has one of the highest cancer mortality rates in the world (1). Despite recent advances in the clinical and experimental fields of HCC, the survival rate of HCC patients is still very low after 5 years $(2,3)$. Most HCC patients are diagnosed in the late stage, by which point treatment options are lacking. Recurrence and metastasis often occur in patients following surgical resection and liver transplantation, and for those who 
do not meet the conditions for surgery, neoadjuvant therapy, chemotherapy, and radiation therapies have made outstanding contributions to the treatment of HCC. Some other treatment methods still require validation through clinical trials, and most of these therapies have the disadvantage of obvious side effects $(4,5)$. In order to improve the outcomes of HCC patients, it is necessary to find biomarkers for early diagnosis of HCC, as well as new therapeutic targets affecting the prognosis and prescription of potential treatments for HCC.

Non-coding RNA (ncRNA) refers to RNA that does not code for protein, accounting for more than $90 \%$ of total human RNA (6). Previous studies have shown that ncRNA is involved in the process of a variety of tumors (7). To date, HCC-specific microRNAs (miRNA) such as miR122 have been screened as potential therapeutic targets, but there are still many HCC-related miRNAs awaiting exploration (8). Recently, a kind of non-coding circular RNA (circRNA) has attracted increasing attention and research. According to reports, it can act as a sponge for miRNA, thereby participating in a series of biological and pathological processes. It has the stability messenger RNA (mRNA) and other ncRNAs do not possess, and is not easily degraded by RNase (9). CircRNA can play a regulatory role in a variety of ways. First of all, circRNA can spongelike adsorption of miRNA that binds to its miRNA binding site. The latter can affect cell metabolism by regulating the expression and activity of target proteins. Secondly, circRNA regulates alternative splicing or transcription of RNA, including competition with linear RNA in the splicing link of precursor mRNA and the interaction with RNA polymerase II complex to promote the transcription of circRNA parent genes. Furthermore, circRNA may interact with RNA-binding protein to regulate protein translation efficiency. In addition, a small number of cyclic RNAs have translation functions and can encode short peptides with biological activity (10-13). Therefore, it is of great significance to explore HCC-related circRNA and its mechanism of action in HCC, investigate its potential as an early diagnostic marker of HCC, investigate potential prognostic indications, and whether it can potentially serve as a new target for treatment.

At present, the drug resistance of targeted therapy for liver cancer is a major difficulty. As a classic drug, drug resistance is an important factor affecting its later treatment. Luo et al. (14) found that in cisplatin-resistant liver cancer tissues and cell lines, the expression of a new circRNA_101505 was reduced, and it was associated with a poor prognosis of liver cancer; further studies found that overexpression of circRNA_101505 can inhibit liver cancer cells The growth of cisplatin enhances the toxic effect of cisplatin on liver cancer cells. The mechanism is that circRNA_101505 sensitizes liver cancer cells to cisplatin through sponge miR-103, thereby promoting the expression of oxidized nitro-domain-containing protein 1 . With the in-depth research on specific circRNA in HCC, its related signal pathways and mechanism of action involved in the occurrence and development of liver cancer will be revealed one by one, so as to provide more theoretical basis for the development of specific molecular targeted drugs.

In our research, we utilized the bioinformatics software circBase (http://www.circbase.org/) to discover the unresearched circ_NOTCH3, which is related to HCC cell lines. According to reports, circ_NOTCH3 is a protooncogene and a powerful biomarker that can act as a sponge, compete with miR-205-5p, regulate the expression of KLF12, and promote the development and progress of basal-like breast carcinoma. However, the role of circ NOTCH3 in HCC has not been reported (15). First, we measured circ_NOTCH3 in the database and compared it with the expression outcomes in HCC cell lines, normal liver cell lines, cancer specimens, and paracancerous specimen samples. The outcomes showed circ_NOTCH3 is highly expressed in HCC. After transfecting small interfering RNA (siRNA) into HUH7 and HepG2 cell lines, we discovered circ_NOTCH3 boosted the migration of HCC cells. We also implemented bioinformatics software to predict potential miRNA targets with circ_NOTCH3 binding sites, and specifically identified miR-875-5p.

A molecular biology experiment verified circ_NOTCH3 promotes ZNF146 by competitively binding miR-875-5p, indicating circ_NOTCH3 as a potential target for HCC treatment. We present the following article in accordance with the ARRIVE reporting checklist (available at https:// dx.doi.org/10.21037/jgo-21-567).

\section{Methods}

\section{Collection of HCC specimens}

From February 2020 to January 2021, 30 patients undergoing liver cancer resection at Yantai Yuhuangding Hospital were collected for study. The inclusion criteria are as follows: (I) HCC patients diagnosed pathologically; (II) patients undergoing radical surgical resection; (III) age >18 years; (IV) complete case data and postoperative follow-up data. 
For each patient included in the study, 20 pairs of matched HCC cancer tissues and corresponding distal nontumor tissues (also known as adjacent tissues should be at least distance from the tumor) without affecting the tissue required for pathological diagnosis after surgical resection ( $5 \mathrm{~cm}$, as a control), cut the liver tissue into mung bean to soybean grain size tissue pieces, put them into labeled cryotubes, record the corresponding patient information, quickly put them in liquid nitrogen and freeze them, and store them in-stand-by in $80{ }^{\circ} \mathrm{C}$ ultra-low temperature refrigerator.

All procedures performed in this study involving human participants were in accordance with the Declaration of Helsinki (as revised in 2013). The study was approved by ethics board of Qingdao No. 6 People's Hospital (No. 2020003 ) and informed consent was taken from all patients.

\section{Cell lines and transfection}

Normal human liver cells LO2 and HCC cells (HUH7, HepG2, 7721, together with LM3) were inoculated in Roswell Park Memorial Institute (RPMI) 1640 medium [containing $100 \mathrm{~mL} / \mathrm{L}$ fetal bovine serum (FBS), $100 \mathrm{IU} / \mathrm{mL}$ penicillin, $100 \mu \mathrm{g} / \mathrm{mL}$ streptomycin], and cultured in a $37^{\circ} \mathrm{C}$ $\mathrm{CO}_{2}$ incubator. When the cell confluence reached $70 \%$, the instructions of Lipofectamine ${ }^{\mathrm{TM}} 3000$ Transfection Reagent (Thermo Fisher Scientific, Waltham, MA, USA) were followed for transfection. Lipofectamine ${ }^{\mathrm{TM}} 3000$, shcirc_NOTCH3, pcDNA3.1-circ_NOTCH3, miR-875-5p mimics, and pcDNA3.1-ZNF146 were diluted, respectively. The $\mathrm{P} 3000^{\mathrm{TM}}$ reagent was added to the above DNA diluents and mixed thoroughly. Then, the diluted Lipofectamine ${ }^{\mathrm{TM}}$ 3000 was added at a ratio of $1: 1$, and incubated at room temperature for 10-15 min. Subsequently, the DNAlipid complex was added to HUH7 cells, and the final transfection volume was: DNA $2.5 \mu \mathrm{g}, \mathrm{P} 3000^{\mathrm{TM}} 5 \mu \mathrm{L}$, and Lipofectamine $^{\mathrm{TM}} 30003.75 \mu \mathrm{L}$. After $48 \mathrm{~h}$ of culture, realtime fluorescence quantitative polymerase chain reaction (PCR) and western blot were employed to measure the levels of circ_NOTCH3, miR-875-5p as well as ZNF146.

\section{Reverse transcription-PCR (RT-PCR) assay}

TRIzol reagent was implemented to extract total RNA from HCC specimens, adjacent specimens, and HCC cells, and a one-step RT kit was used to reverse all RNA into complementary DNA (cDNA). TaqMan Universal PCR Master Mix kit instructions were used for PCR reaction.
The reaction system was: Taqman Master mix $10 \mu \mathrm{L}$, diethyl pyrocarbonate (DEPC) water $7.67 \mu \mathrm{L}$, TaqMan RNA assay mix $1 \mu \mathrm{L}$, and cDNA $1.33 \mu \mathrm{L}$, to a total of $20 \mu \mathrm{L}$. The reaction program was as follows: $95{ }^{\circ} \mathrm{C}$ pre-denaturation $5 \mathrm{~min}$, denaturation at $95^{\circ} \mathrm{C}$ for $15 \mathrm{~s}$, annealing at $60{ }^{\circ} \mathrm{C}$ for $30 \mathrm{~s}$, and extension at $72{ }^{\circ} \mathrm{C}$ for $15 \mathrm{~s}$, for a total of 50 cycles. We implemented $2^{-\Delta \Delta C t}$ to calculate the relative quantitative outcomes, and was used U6 as the miRNA internal reference. The relative expression of mRNA and circRNA was based on the expression of GAPDH in the same sample as an internal control. The circ_NOTCH3 upstream primer was 5'-TATGTTGGTGGATCCTGTTCGGCA-3', downstream primer was 5'-TGGTGGGTAGAC CAAGACTTGTGA-3'; miR-875-5p upstream primer was 5'-GGACTTTCTTCATTCACACCG-3', downstream primer was 5'-GACCACTGAGGTTAGAGCCA-3'; ZNF146 upstream primer was 5'-CTCGCTTCGGC AGCACATATACT-3', and the downstream primer was 5'-ACGCTTCACGAATTTGCGTGTC-3'.

\section{Western blot}

We transfected sh-circ_NOTCH3 and miR-875-5p mimics alone, or co-transfected pcDNA3.1-circ_NOTCH3 and miR-875-5p mimics, miR-875-5p mimics and pcDNA3.1-ZNF146, sh-circ_NOTCH3 and pcDNA3.1ZNF146. After $48 \mathrm{~h}$ of treatment, the cells of each group were collected. In each group, the same amount of protein samples were employed for sodium dodecyl sulfate-polyacrylamide gel electrophoresis (SDS-PAGE) separation, and the target protein bands were transferred to the polyvinylidene fluoride (PVDF) membrane by electroporation. After blocking with skimmed milk powder at room temperature for $1 \mathrm{~h}$, we added rabbit antiZNF146 antibody (abcam, 1:1,000; Abcam, Cambridge, MA, USA), and rabbit anti-GAPDH antibody (abcam, $1: 10,000)$, respectively, at $4^{\circ} \mathrm{C}$ overnight. After washing, the corresponding horseradish peroxidase (HRP)-labeled goat anti-rabbit IgG $(1: 5,000)$ was added and incubated at $37^{\circ} \mathrm{C}$ for $1 \mathrm{~h}$. Then, enhanced chemiluminescence (ECL) reagent was used to develop color. Image J software (https://imagej. nih.gov/ij/) was used to analyze the band absorbance value.

\section{3-(4,5-dimethylthiazolyl-2)-2,5-diphenyltetrazolium bromide (MTT) assay}

Transfection of circ_NOTCH3 overexpression and its empty control lentiviral vector into hepatoma cell line 
HUH7 was conducted according to the specific transfection method. The cells were cultured in 96-well culture plates with a density of $5 \times 10^{3} /$ well. After the cells adhered to the wall for $48 \mathrm{~h}$, the cell proliferation activity was analyzed every $24 \mathrm{~h}$ with $20 \mu \mathrm{L}$ of MTT $(5 \mathrm{mg} / \mathrm{mL})$ and incubated for $4 \mathrm{~h}$. The absorbance value at $570 \mathrm{~nm}$ was tested with a microplate reader. The experiment was divided into circ_ NOTCH3 group and CON group, repeated 3 times.

\section{Colony formation assay}

The HCC cells growing in logarithmic phase were taken and counted as $1 \times 10^{3}$ cells per well. After counting, the cells were seeded in a 6 -well cell culture plate and cultured until cell colonies were formed. The experiment was divided into empty vector negative control group and circ_NOTCH3 overexpression group. After transfecting the overexpression vector and the empty vector into the cells and culturing for $48 \mathrm{~h}$, the culture medium was discarded, and the cells were fixed with $40 \mathrm{~g} / \mathrm{L}$ paraformaldehyde for $15 \mathrm{~min}$. Then, the paraformaldehyde was discarded and the cells washed, $1 \mathrm{~g} / \mathrm{L}$ crystal violet was added at room temperature, the dye solution was washed off with phosphate-buffered saline (PBS), and the number of colonies formed was observed through a microscope.

\section{Cell apoptosis analysis}

When the cells reached $50 \%$ confluence, the overexpression vector and the empty vector were transfected to the cells and cultured for $48 \mathrm{~h}$, then the Hoechst 33258 kit was used to stain the cells according to the instructions. At the same time, the cells were digested with $2.5 \mathrm{~g} / \mathrm{L}$ trypsin, and then washed twice with Dulbecco's PBS (DPBS), and centrifuged at 2,000 r/min for $5 \mathrm{~min} ; 500 \mu \mathrm{L}$ of buffer was added to the cell pellet to resuspend the cells, $5 \mu \mathrm{L}$ annexin $\mathrm{V}$-APC and $10 \mu \mathrm{L}$ propidium iodide (PI) was added and mixed well. This was incubated for $15 \mathrm{~min}$ at room temperature in the dark, and cell apoptosis was detected by flow cytometry.

\section{Transwell assay}

The overexpressed circ_NOTCH3 and its empty control lentiviral vector were transfected into the hepatoma cell line HUH7. After the cells had adhered to the wall for $48 \mathrm{~h}$, they were treated with $0.5 \mu \mathrm{mol} / \mathrm{L}$ oxaliplatin for $24 \mathrm{~h}$. Then, the cells were suspended in a serum-free medium and starved for $24 \mathrm{~h}$, the suspended cells were added to the upper chamber containing matrix gel $(3.9 \mu \mathrm{g} / \mu \mathrm{L})$; and the lower chamber contained RPMI-1640 medium with $10 \%$ FBS. Finally, the cells passing through the gel were fixed with $75 \%$ ethanol and stained with crystal violet. Under an optical microscope, three fields of view were selected for cell count in each group. The experiment was divided into circ_NOTCH3 + oxaliplatin group, $\mathrm{CON}+$ oxaliplatin group, and CON + PBS group, and performed in triplicate.

\section{Dual luciferase reporter assay}

The StarBase website (http://starbase. sysu.edu.cn/) was used to predict the binding sequence of miR-875-5p with circ_NOTCH3 and ZNF146, and introduce the binding sequence of circ_NOTCH3 and ZNF146 with miR-875-5p into the dual-luciferase reporter gene vector, respectively, to construct pmiR-Report-circ_NOTCH3 and pmiR-ReportZNF146 wild type (WT) vector WT-circ_NOTCH3 and WT-ZNF146. We used gene-directed mutagenesis technology to mutate the sequence of circ_NOTCH3 and ZNF146 and introduce the double luciferase reporter gene vector to construct mutant type (MUT) vector MUTcirc_NOTCH3, MUT-ZNF146. The pmiR-Report-circ_ NOTCH3 and pmiR-Report-ZNF146 WT or mutant vectors were transfected alone or separately with miR875-5p mimics into HEK $293 \mathrm{~T}$ cells. After culturing for $48 \mathrm{~h}$, the dual luciferase reporter gene kit was used to detect fluorescence enzyme activity.

\section{Hematoxylin and eosin (HE) staining}

After deparaffinization and rehydration, $5 \mu \mathrm{m}$ tissue sections were stained with hematoxylin solution for $5 \mathrm{~min}$. The sections were soaked in $1 \%$ acid ethanol and rinsed in distilled water. Then the sections were stained with eosin solution for $3 \mathrm{~min}$ and followed by dehydration with graded alcohol and clearing in xylene. The mounted slides were then examined and photographed using an Olympus BX53 fluorescence microscope (Tokyo, Japan).

\section{IHC}

After deparaffinization and rehydration, the slices were sealed with $10 \%$ BSA (Bovine Serum Albumin) at room temperature for half an hour. The slices were then incubated overnight with primary antibody (PA5-51343, Thermo Fisher Scientific, New York, USA) in a 4-degree refrigerator. The next day, the slides were taken out and 
incubated with secondary antibody in a $37^{\circ} \mathrm{C}$ incubator for half an hour. Wash 3 times in PBS buffer for 5 minutes each time. Then the slides were stained according to instruction of 3,3'-diaminobenzidine (DAB) substrate kit (Abcam, Cambridge, UK). The slides were then photographed using an Olympus BX53 fluorescence microscope (Tokyo, Japan). Immunohistochemical analysis of paraffin-embedded tissue employed ZNF146 antibody (Proteintech,20385-1-AP) at dilution of 1:100.

\section{In vivo tumor growth assay}

BALB/c nude mice, male, 4-6 weeks, body weight 18-20 g, were randomly divided into si-NC and siRNA pool group, 6 mice in each group. After si-NC and siRNA pool were transfected into hepatoma cell line HUH7, subcutaneous injection $\left(1 \times 10^{7} /\right.$ mouse $)$ was administered to establish a nude mouse subcutaneous xenograft animal model. The long diameter (a) and short diameter (b) were measured of the subcutaneous tumor in nude mice with a vernier caliper every other day, and the tumor volume was calculated $\left(\mathrm{V}=1 / 2 \times a \times b^{2}\right)$. Nude mice in each group were sacrificed 32 days after injection. Experiments were performed under a project license (No. 2020-011) granted by ethics board of Qingdao No. 6 People's Hospital, in compliance with NIH guidelines for the care and use of animals.

\section{Statistical analysis}

The software SPSS 20.0 (IBM Corp., Armonk, NY, USA) was used for statistical analysis and quantitative data were expressed as mean \pm standard deviation $\left(\bar{x}_{ \pm} \mathrm{S}\right)$. The independent sample $t$-test was employed for comparison between the two groups of data, and one-way analysis of variance (ANOVA) was employed for the comparison between the two groups of data. Statistical significance was considered when $\mathrm{P}<0.05$.

\section{Results}

\section{Circ_NOTCH3 is elevated in $\mathrm{HCC}$}

To identify the characteristics of circ_NOTCH3, specific experiments exhibited that circ_NOTCH3 was resistant to RNase $\mathrm{R}$ treatment instead of the linear subtype (Figure 1A). Fluorescence in situ hybridization (FISH) experiments unveiled that circ_NOTCH3 is mainly located in the cytoplasm, while its linear subtype was located in both the nucleus and cytoplasm (Figure $1 B$ ). We detected the circ NOTCH3 in HUH7 and Hepg2 cells and found that the transfection rate of siRNA-circ_NOTCH3 was effective (Figure 1C). Subsequently, we discovered that compared with the healthy human liver cell line LO2, circ_NOTCH3 in the four HCC cell lines was obviously augmented (Figure 1D). We further verified that circ_NOTCH3 was up-regulated in HCC specimen samples compared with normal samples (Figure 1E). Additionally, we discovered circ_NOTCH3 level in metastatic HCC specimens was higher than that in non-metastatic HCC specimens (Figure $1 F$ ). These outcomes suggest circ_NOTCH3 is elevated in HCC, indicating it may be related to the HCC pathogenesis.

\section{Silencing circ_NOTCH3 restrains the growth of HCC}

In order to study the function of circ_NOTCH3 in HCC, we stably transfected siRNA into HUH7 and HepG2 cells to investigate cell phenotype. The MTT experiments unveiled knocking down circ_NOTCH3 evidently lowered the viability of HUH7 and HepG2 cells (Figure 2A). The outcomes unveiled the colony formation rate of the siRNA group was evidently down-regulated (Figure $2 B$ ). The soft agar colony formation experiment also unveiled the number of colonies of HUH7 and HepG2 cells transfected by the siRNA was suppressed (Figure 2C). Besides, circ_NOTCH3 silencing was shown to cause a sharp augment in $\mathrm{HUH} 7$ and HepG2 cell apoptosis (Figure 2D). In HUH7 and HepG2 cells, transwell together with wound healing experiments were utilized to evaluate the influence of knocking out circ $\mathrm{NOTCH} 3$ on cell migration. The outcomes unveiled that compared with the matched group, the migration ability of HUH7 and HepG2 cells in the siRNA group was obviously restrained (Figure 2E,2F). Additionally, we displayed circ_NOTCH3 knockdown led to an obvious reduction of aggressive HUH7 and HepG2 cells (Figure 2G). In order to explore the effect of circ_NOTCH3 in the resistance of HCC cells, we treated HUH7 together with HepG2 cells with different concentrations of oxaliplatin, and then implemented the MTT method to detect the cell survival rate. In the presence of different concentrations of oxaliplatin, the viability of HUH7 together with HepG2 cells transfected with the siRNA was evidently restrained (Figure 2H). Additionally, we discovered that HUH7 and HepG2 cells treated with the siRNA had an obvious decrease in activity after oxaliplatin treatment (Figure 2I). In conclusion, the silencing of circ_NOTCH3 was shown to 

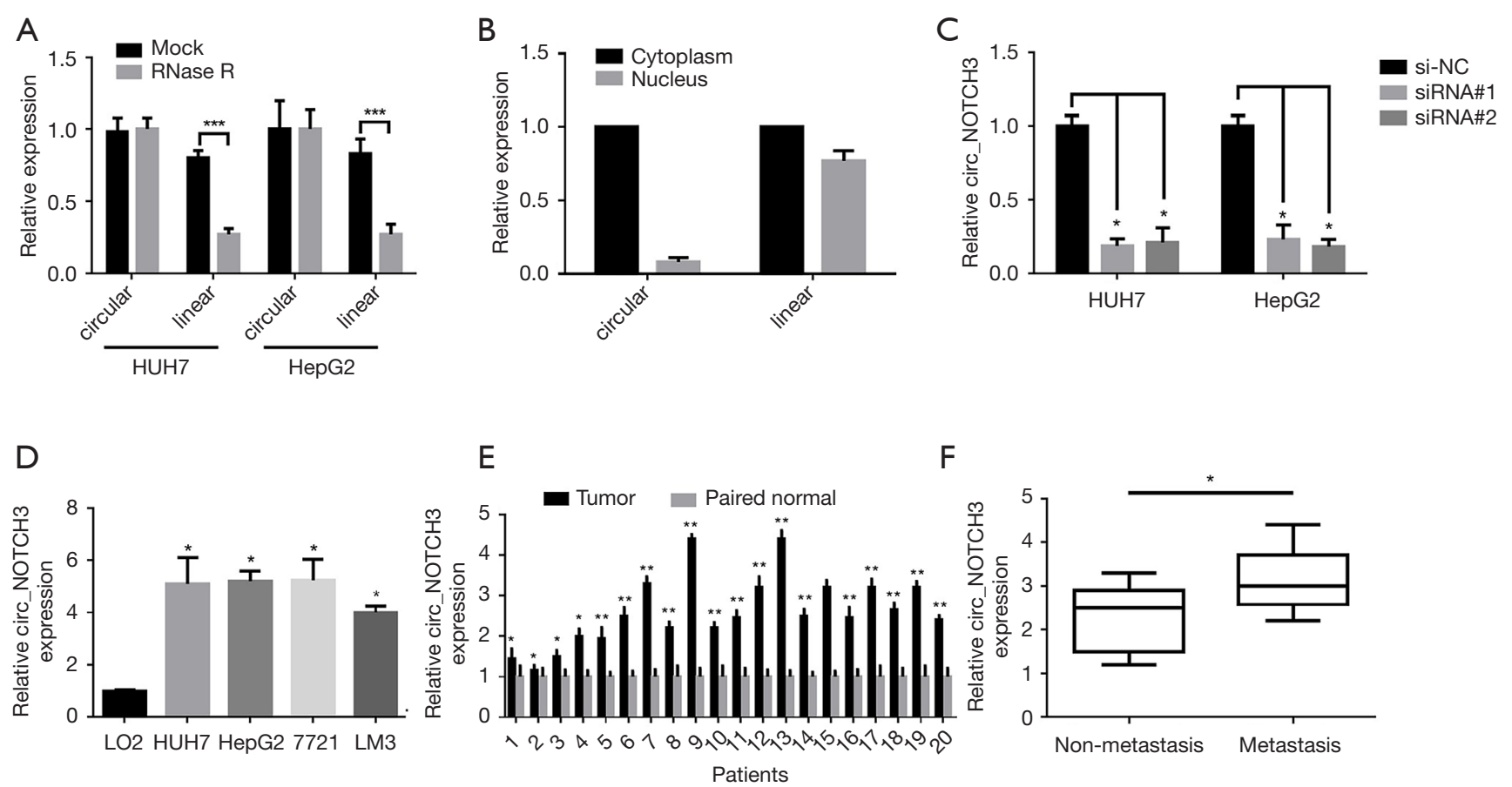

Figure 1 Circ_NOTCH3 was ascertained to be elevated in HCC. (A) In the presence of RNaser, circ_NOTCH3 and linear mRNA were verified by qRT-PCR in HUH7 and HepG2 cells. (B) FISH was utilized to detect the distribution of circ_NOTCH3 in HUH7 cells, and DAPI was implemented to stain the nucleus. (C) Design of two siRNAs to target the reverse splicing connection of circ_NOTCH3, and si-NC as a negative control. (D) Circ_NOTCH3 in HUH7, HepG2, 7721, and LM3 HCC cell lines. The control group was NCM460. (E) QRTPCR was utilized to detect circ_NOTCH3 in HCC and normal specimens. (F) Circ_NOTCH3 in metastatic and non-metastatic HCC specimens. * $\mathrm{P}<0.05 ;{ }^{* *}, \mathrm{P}<0.01$; ${ }^{* *}, \mathrm{P}<0.001$. HCC, hepatocellular carcinoma; mRNA, messenger RNA; qRT-PCR, real-time quantitative reverse transcription polymerase chain reaction; FISH, fluorescence in situ hybridization; DAPI, 4',6-diamidino-2-phenylindole; siRNA, small interfering RNA. si-NC, siRNA-NC.

diminish the growth of HCC cells in vitro.

\section{Circ_NOTCH3 as a sponge for miR-875-5p}

To ascertain whether circ_NOTCH3 can regulate miRNAs in sponge cells, we selected five candidate miRNAs through StarBase (Figure $3 A$ ). Then, we checked in HUH7 and HepG2 cells overexpressing stable circ_NOTCH3, the pull-down efficiency was evidently improved (Figure 3B). After pulling down, the five candidate miRNAs were investigated by real-time quantitative RT-PCR (qRT-PCR). The outcomes unveiled the circ_NOTCH3 probe greatly down-regulated miR-875-5p together with miR-338-3p (Figure 3C,3D). Additionally, we discovered circ_NOTCH3 was obviously up-regulated in $\mathrm{HUH} 7$ and HepG2 cells compared with the cells treated with biotin-labeled miR875-5p MUT (Figure 3E,3F). Furthermore, luciferase reporter gene testing was applied to verify whether miR- 875-5p directly bound with circ_NOTCH3. After HUH7 together with HepG2 cells were co-transfected with miR875-5p mimic together with circ_NOTCH3 WT reporter plasmid, the luciferase level decreased (Figure $3 G, 3 H$ ). These outcomes indicate that circ_NOTCH3 acts as a sponge of miR-875-5p in HCC cells.

\section{Transfection of miR-875-5p suppressed the growth of HCC cells in vitro}

Kaplan-Meier analysis unveiled HCC patients with low expression of miR-875-5p had a poorer prognosis (Figure 4A). By qRT-PCR analysis of miR-875-5p, we discovered miR-875-5p in HCC specimens and cell lines (HUH7, HepG2, 7721 together with LM3) was evidently lower than in normal specimens together with NCM460 cell lines (Figure 4B,4C). Next, we evaluated the influences on overexpression of miR-875-5p on cell proliferation, 
A

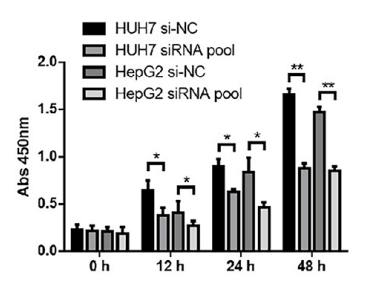

D

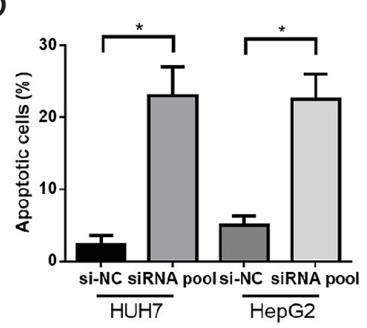

F

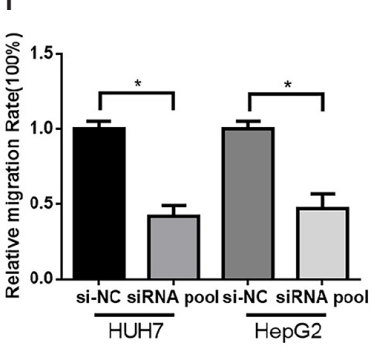

$\mathrm{H}$

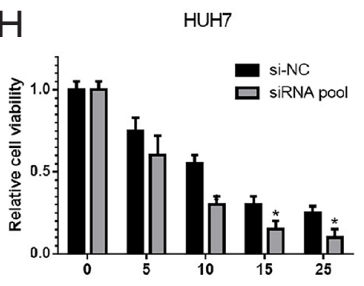

B

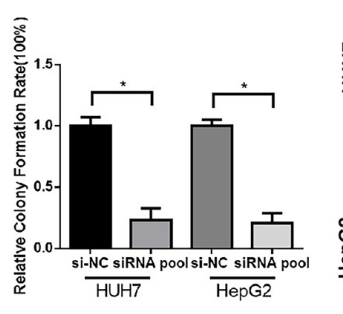

i-NC

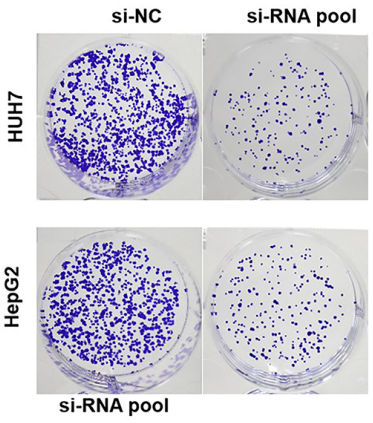

C

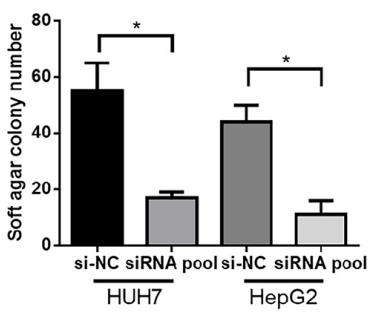

E
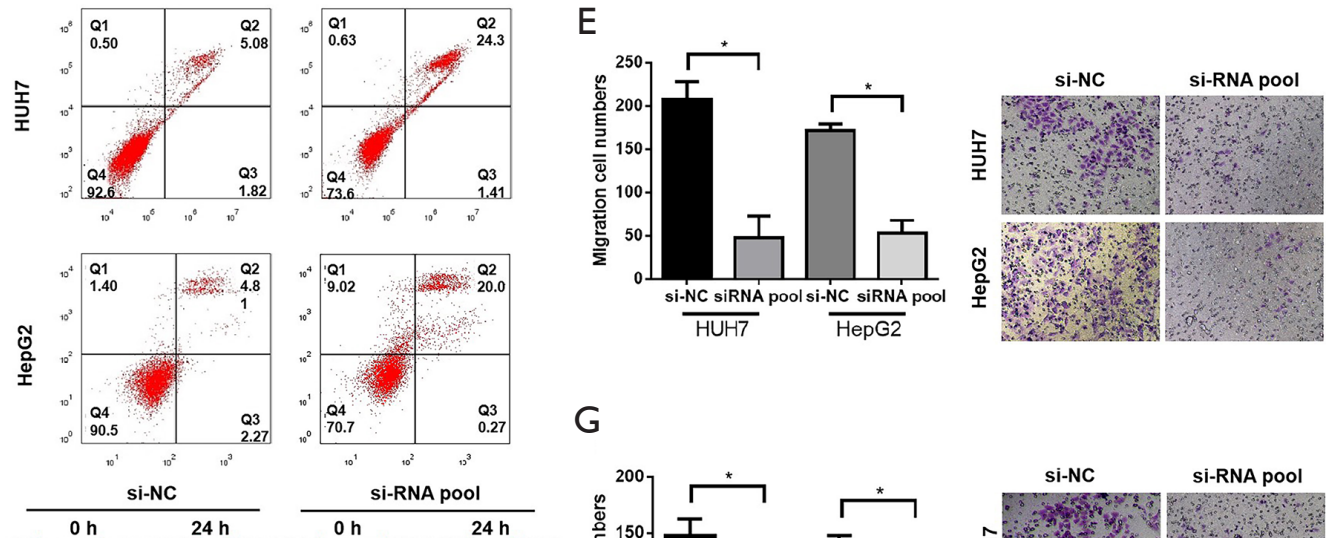

G
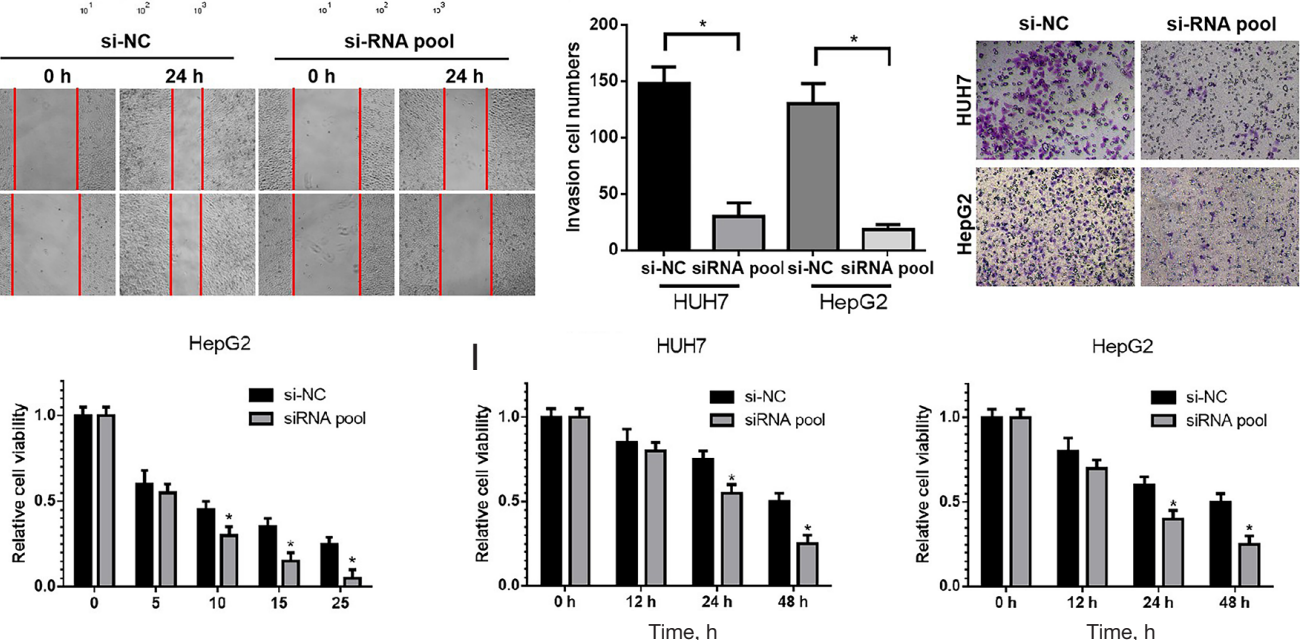

Figure 2 Silencing circ_NOTCH3 suppressed the growth of HCC cells in vitro. (A) After transfection of si-NC and siRNA 0, 24, and 48 h, the viability of HUH7 and HepG2 cells was detected. After $24 \mathrm{~h}$ of transfection of si-NC and siRNA pool, HUH7 and HepG2 cells were subjected to (B) colony formation (1 g/L crystal violet was used to stain cells), (C) soft agar and (D) flow cytometry. (E) Transwell experiment (cells were stained by crystal violet, magnification 200x) and (F) wound healing experiment to detect the migration ability of HUH7 and HepG2 cells transfected with si-NC or siRNA pool. (G) The influence on knocking down circ_NOTCH3 on the invasion ability of HUH7 and HepG2 cells (cells were stained by crystal violet, magnification 200x). (H) MTT was implemented to evaluate the feasibility of circ NOTCH3-silenced HUH7 HepG2 cells treated with different concentrations of oxaliplatin (0, 5, 10, 15, and $25 \mu \mathrm{g} / \mathrm{mL}, 24 \mathrm{~h}$ ). (I) After 0, on the 12th, 24th, the feasibility of $48 \mathrm{~h}$ [oxaliplatin $(5 \mu \mathrm{g} / \mathrm{mL})$ treatment] circ_NOTCH3-silenced HUH7 HepG2 cells receiving oxaliplatin were detected. *, $\mathrm{P}<0.05$; **, $\mathrm{P}<0.01$. HCC, hepatocellular carcinoma; siRNA, small interfering RNA; MTT, 3-(4,5-dimethylthiazolyl-2)2,5-diphenyltetrazolium bromide. 
A

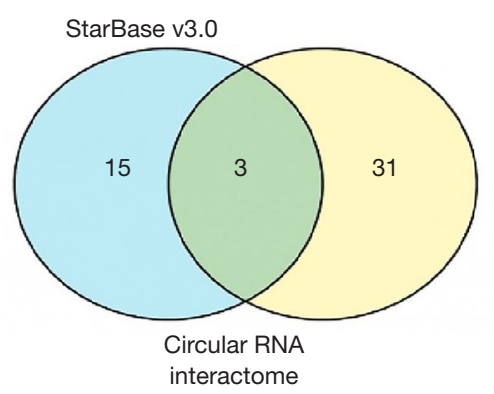

$\mathrm{D}$

.

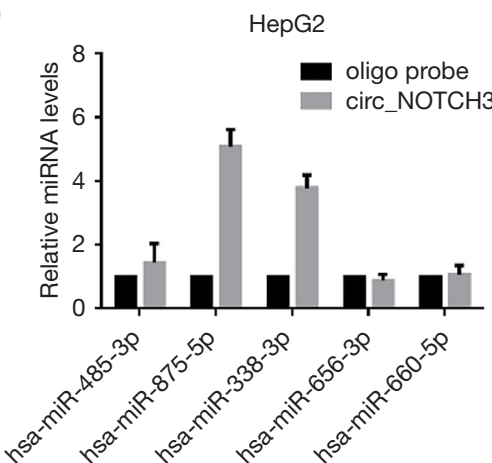

G

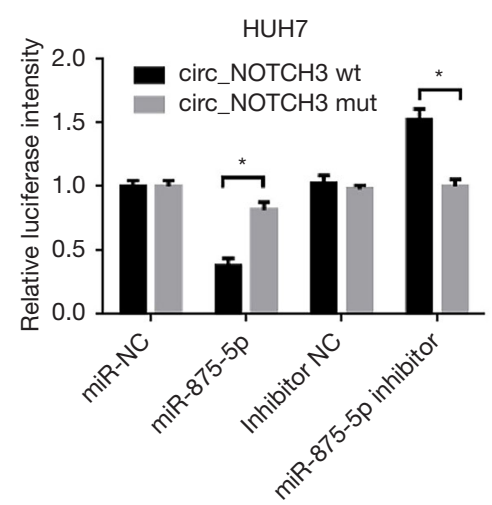

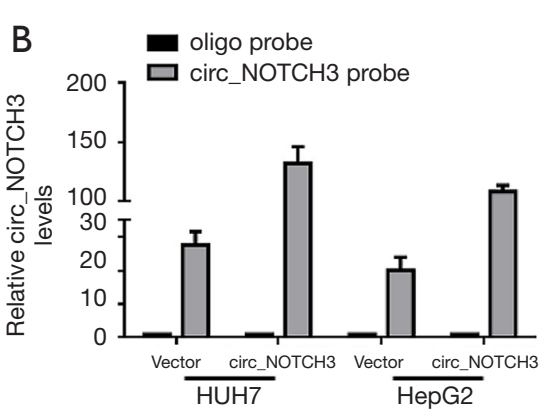

$\mathrm{E}$

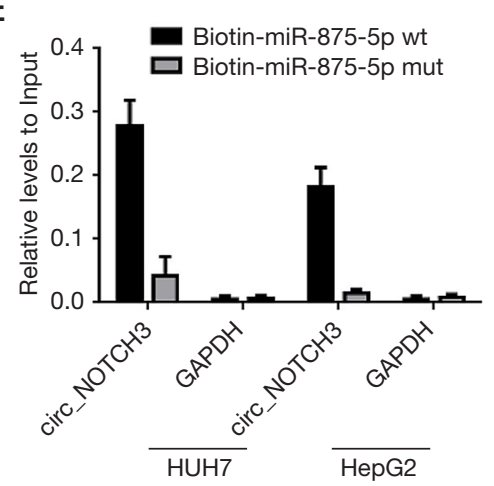

$\mathrm{H}$

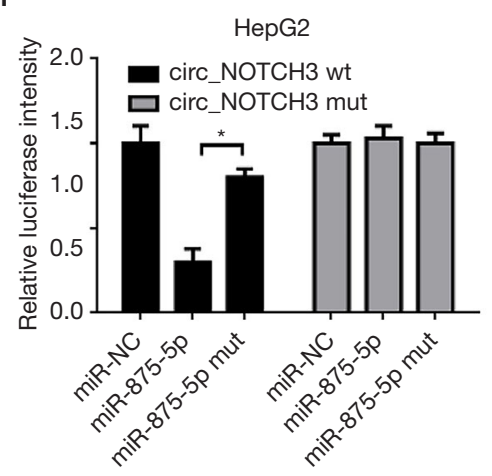

Figure 3 Circ_NOTCH3 acts as a sponge for miR-875-5p. (A) StarBase (v3.0) and circRNA interactome predict the target miRNAs of circ_NOTCH3. (B) Specific circ_NOTCH3 probes were used to pull down the vector or circ_NOTCH3 transfected HUH7 and HepG2 cell lysates, and then RT-PCR and qRT-PCR were performed. (C,D) Circ_NOTCH3 probe or oligo probe down-regulates the relative relationship of miR-485-3p, miR-875-5p, miR-338-3p, miR-656-3p, and miR-660-5p in HUH7 and HepG2 lysates. (E) The expression level of circ_NOTCH3 in HUH7 and HepG2 cells captured by biotin-miR-875-5p WT or biotin-miR-875-5p MUT. (F) Schematic diagram of circ_NOTCH3-WT and circ_NOTCH3-MUT luciferase reporter vector. (G,H) Luciferase reporter gene detection was performed in HUH7 and HepG2 cells to verify the association between circ_NOTCH3 and miR-875-5p. *, P<0.05. CircRNA, circular RNA; RT-PCR, reverse transcription polymerase chain reaction; qRT-PCR, real-time quantitative RT-PCR; WT, wild type; MUT, mutant type.

invasion, and migration. In the colony formation experiment, we observed that miR-875-5p transfection evidently restrained the colony formation rate of HUH7 and HepG2 cells (Figure 4D). Similarly, transfection of miR-
$875-5 \mathrm{p}$ also led to a decrease in the number of soft agar in HUH7 and HepG2 cells (Figure 4E). In the transwell experiment, we discovered compared with transfected miR-NC, transfected miR-875-5p can obviously diminish 
A
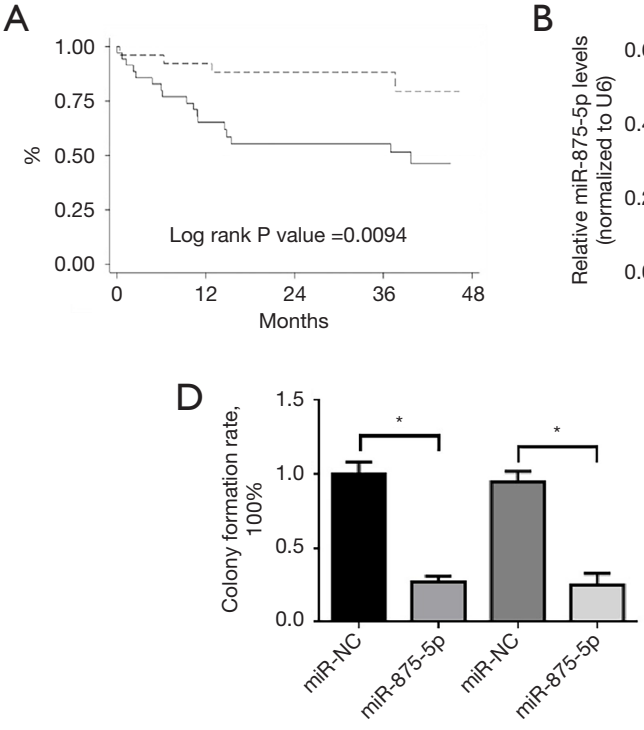

F
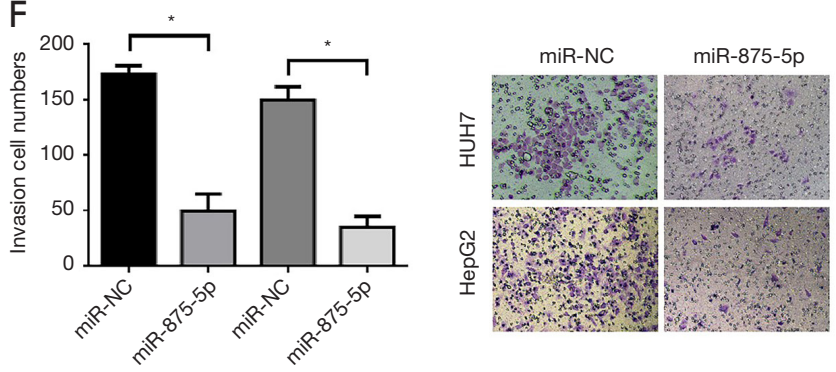

G
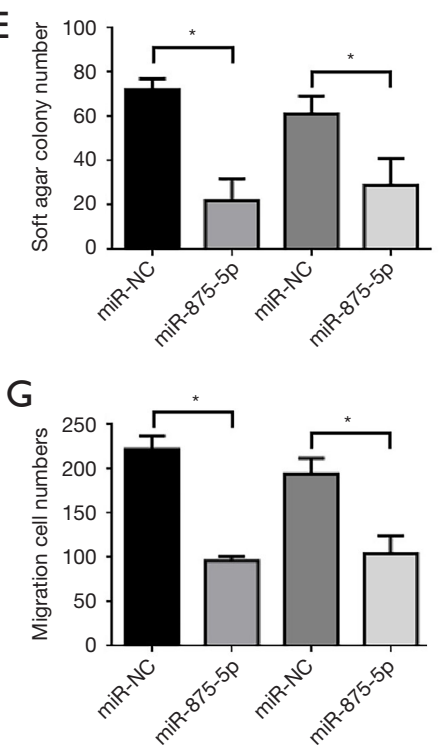

B
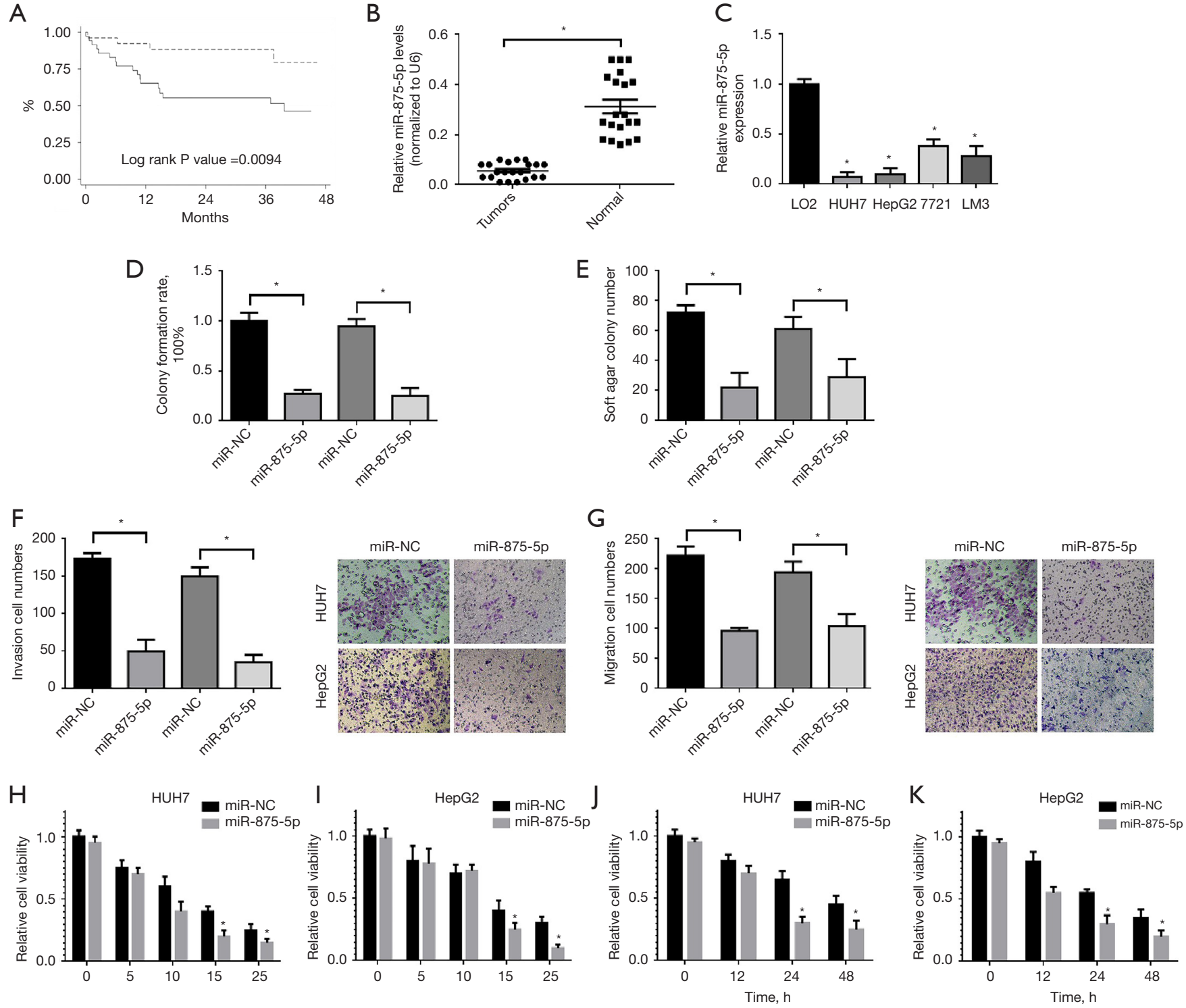

Figure 4 Transfection of miR-875-5p diminishes the growth of HCC cells in vitro. (A) Kaplan-Meier analysis of the prognosis of HCC patients with high or low miR-875-5p expression. (B) The relative expression level of miR-875-5p in HCC specimens and normal specimens. (C) Relative expression levels of miR-875-5p in HCC HUH7, HepG2, 7721, and LM3 cell lines. NCM460 was a control. After miR-NC and miR-875-5p mock transfection for $24 \mathrm{~h}$, the proliferation, invasion, and migration of HUH7 and HepG2 cells were investigated by (D) colony formation, (E) soft agar, and (F,G) transwell assay (cells were stained by crystal violet, magnification 200x). (H,I) To detect the viability of HUH7 and HepG2 cells transfected with miR-875-5p or miR-NC at different concentrations of oxaliplatin $(0,5$, 10, 15, and $25 \mu \mathrm{g} / \mathrm{mL}$ ). (J,K) The viability of HUH7 and HepG2 cells treated with miR-875-5p or miR-NC was measured after $0,12,24$, and $48 \mathrm{~h}$ following treatment with oxaliplatin $(5 \mu \mathrm{g} / \mathrm{mL}) . *, \mathrm{P}<0.05$. HCC, hepatocellular carcinoma.

the invasion and migration ability of HUH7 and HepG2 cells (Figure 4F,4G). Additionally, we also studied the role of miR-875-5p in the resistance of HCC cells to oxaliplatin. The outcomes unveiled that transfection of
miR-875-5p evidently augmented the oxaliplatin sensitivity of HUH7 and HepG2 cells (Figure 4H,4I). Additionally, the study revealed that overexpression of miR-875-5p can evidently reduce the viability of HUH7 and HepG2 
A

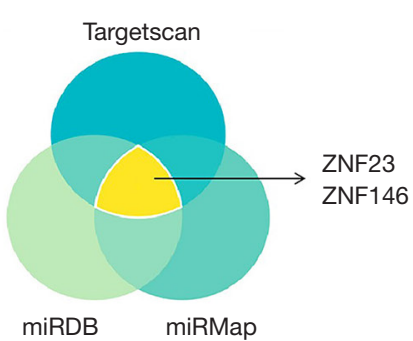

D
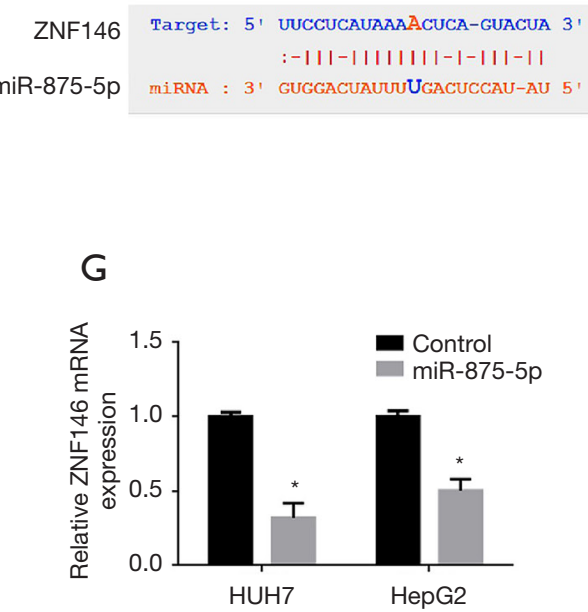

B

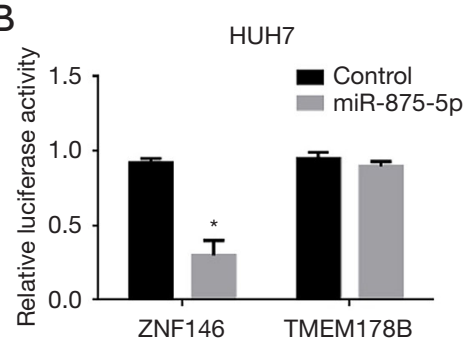

$\mathrm{E}$
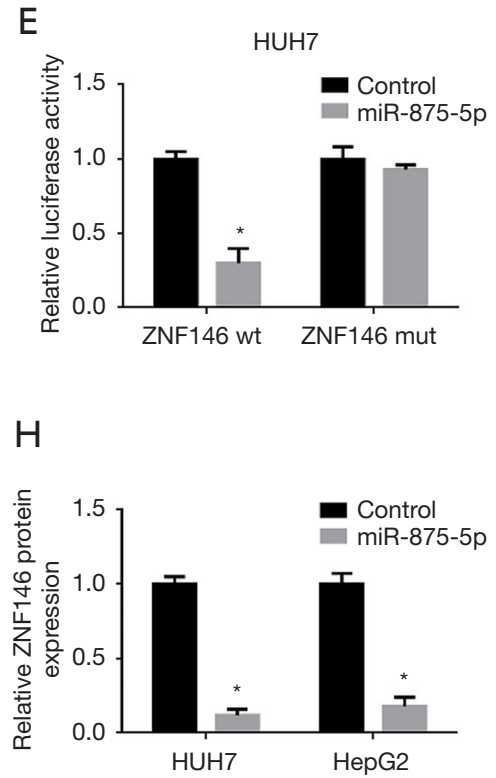

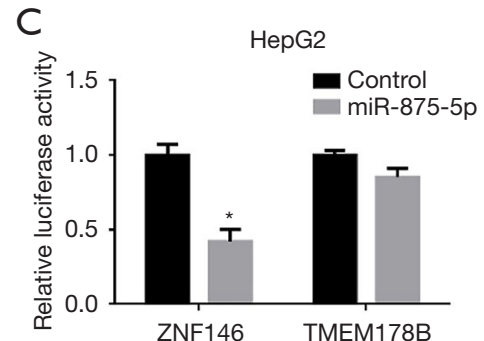

$\mathrm{F}$
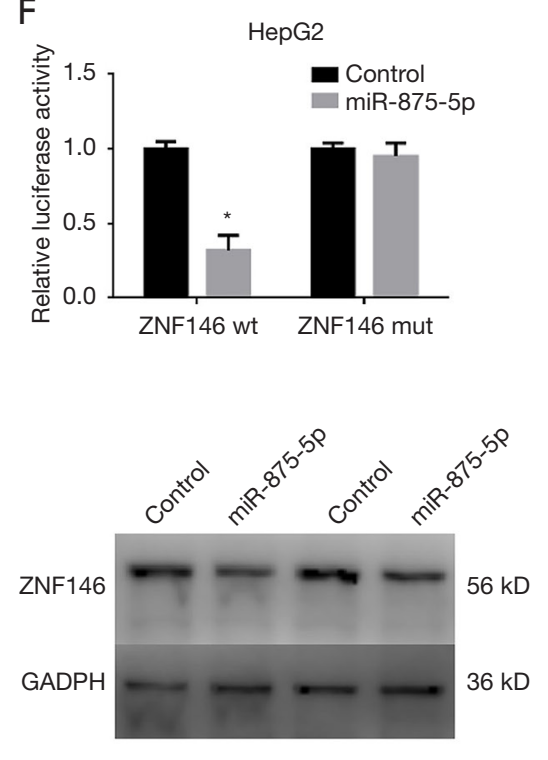

Figure 5 MiR-875-5p diminishes ZNF146 expression by targeting. (A) TargetScan, miRDB, and miRMap to identify the target gene of miR-875-5p. (B,C) The association between miR-875-5p and ZNF146 or ZNF23 was measured by dual luciferase reporter gene method. (D) Schematic diagram of ZNF146-WT and ZNF146-MUT luciferase reporter vector. (E,F) Luciferase reporter gene verification in HUH7 and HepG2 cells co-transfected with miR-875-5p mimic and ZNF146-WT or ZNF146-MUT plasmid. (G,H) Relative expression of ZNF146 mRNA and protein in HUH7 and HepG2 cells transfected with miR-NC or miR-875-5p. *, P<0.05. WT, wild type; MUT, mutant type; mRNA, messenger RNA.

cells after treatment with $5 \mu \mathrm{g} / \mathrm{mL}$ oxaliplatin for 24 and $48 \mathrm{~h}$ (Figure $47,4 \mathrm{~K}$ ). The above outcomes indicate that overexpression of miR-875-5p evidently suppressed the growth of HCC cells in vitro.

\section{MiR-875-5p diminishs its expression by directly binding to ZNF146}

In order to ascertain the target genes of miR-875-5p in HCC cells, we selected ZNF146 as well as ZNF23 through the overlap of database analysis outcomes (Figure $5 \mathrm{~A}$ ). To confirm whether miR-875-5p interacts with these two candidate genes, we performed dual detection of the luciferase reporter gene in $\mathrm{HUH} 7$ as well as HepG2 cells.
The outcomes unveiled that the luciferase level of HCC cells co-transfected with miR-487a-3p and ZNF146 plasmid was obviously lowered, while the luciferase level was not evidently lowered after ZNF23 plasmid transfection (Figure 5B,5C). We further verified the association between miR-875-5p and ZNF146 with ZNF146 WT as well as ZNF146 MUT reporter plasmids (Figure 5D). After transfection of miR-875$5 \mathrm{p}$, the luciferase level of HUH7 and HepG2 cells driven by ZNF146 WT was evidently lowered, while after transfection of miR-875-5p, the luciferase level of ZNF146 MUT was not affected (Figure 5E,5F). Additionally, in HUH7 and HepG2 cells overexpressing miR-875-5p, ZNF146 mRNA as well as protein levels were obviously down-regulated (Figure 5G,5H). The above outcomes indicate miR-875-5p can diminish 

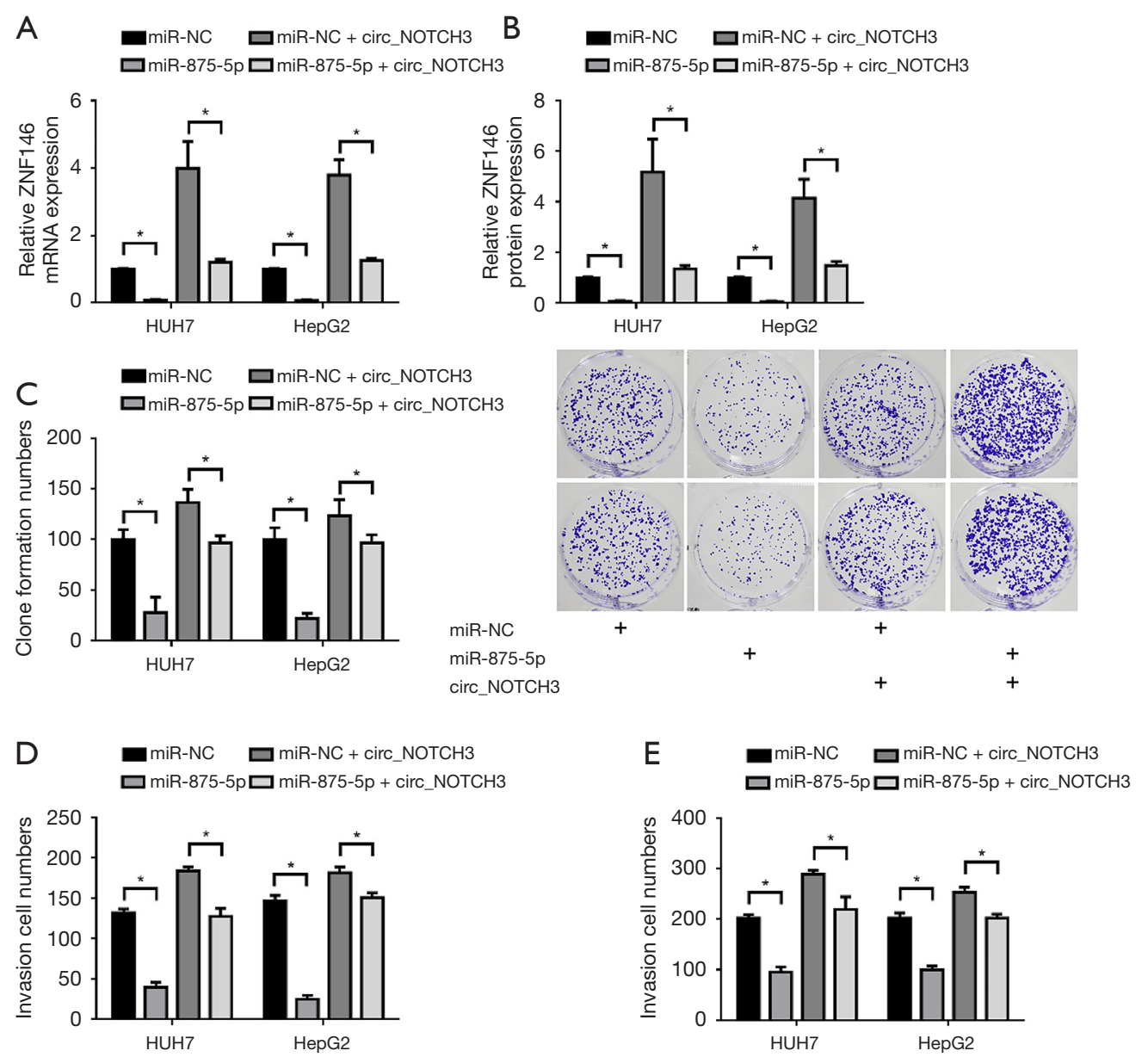

Figure 6 Circ_NOTCH3 eliminates the diminishing influence on miR-875-5p on HCC. (A) Twenty-four hours after miR-875-5p alone or co-transfected with circ_NOTCH3 in HUH7 and HepG2 cells, the relative mRNA levels (B) of ZNF146 were detected. HUH7 and HepG2 cells were transfected with miR-NC, miR-875-5p, miR-NC + circ_NOTCH3, or miR-875-5p + circ_NOTCH3, respectively. After $24 \mathrm{~h}$ of transfection, these cells were subjected to (C) clone formation experiment ( $1 \mathrm{~g} / \mathrm{L}$ crystal violet was used to stain cells), (D) cell invasion and (E) cell migration tests. *, $\mathrm{P}<0.05$. mRNA, messenger RNA;

ZNF146 in HUH7 and HepG2 cells by directly binding to ZNF146.

\section{Circ_NOTCH3 triggers the occurrence of liver cancer by absorbing multiple miRNA}

Since circ_NOTCH3 and miR-875-5p are related in HCC, we next investigated whether circ_NOTCH3 can eliminate the diminishing influence on miR-875-5p on ZNF146 expression as well as HCC cell malignant progression. The outcomes of qRT-PCR and western blot experiments unveiled co-transfection of miR-875-5p and circ NOTCH3 in HUH7 and HepG2 cells can eliminate the downregulation of ZNF146 mRNA and protein induced by miR-875-5p (Figure $6 A, 6 B$ ). In the colony formation experiment, we observed in HUH7 as well as HepG2 cells that the ectopic expression of circ_NOTCH3 restored the cell proliferation activity inhibited by miR-875-5p (Figure 6C). Additionally, through transwell experiments, we discovered that transfection of circ_NOTCH3 in HUH7 along with HepG2 cells can restore cell invasion and migration capabilities, and these capabilities are suppressed by overexpression of miR-875-5p (Figure 6D,6E). These outcomes indicate that circ_NOTCH3 can regulate the proliferation, migration, and invasion of HCC cells through sponge cell miR-875-5p. 
A

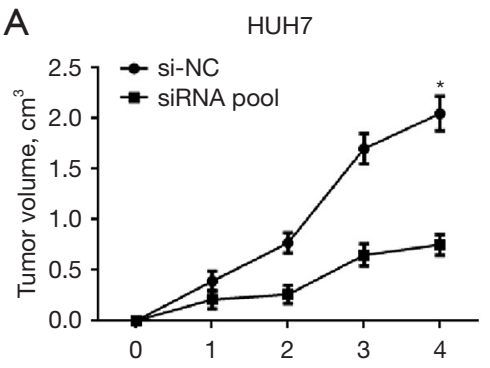

B

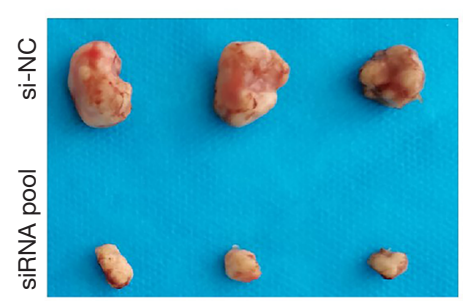

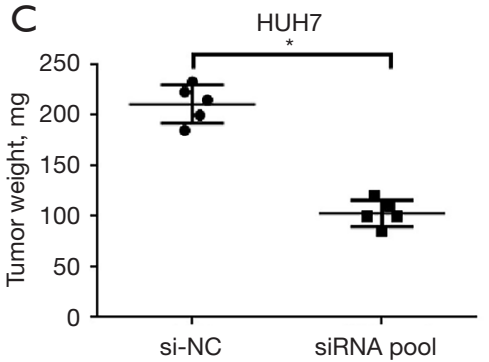

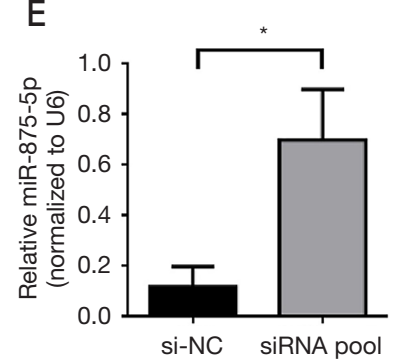

$\mathrm{F}$

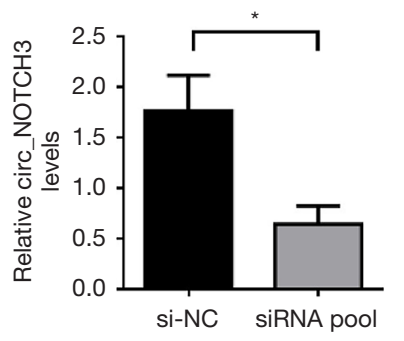

G

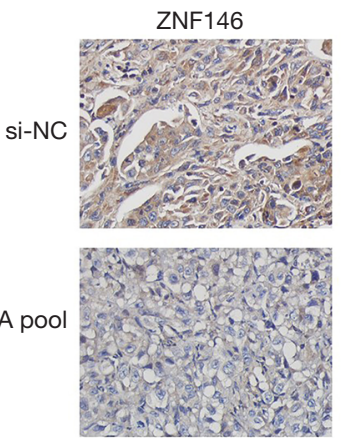

$\mathrm{H}$
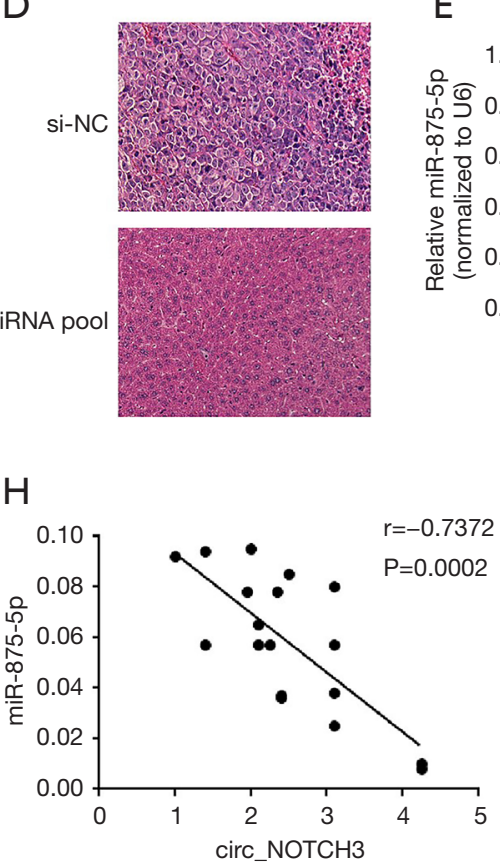

I

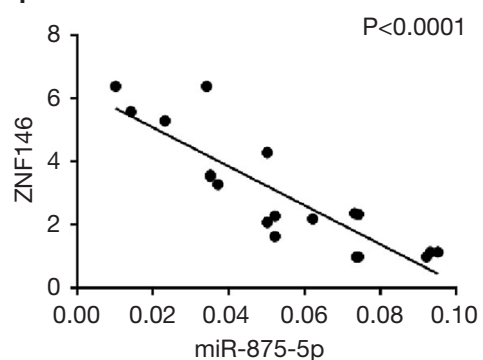

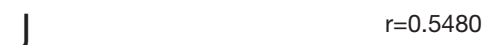

$8 \quad \mathrm{P}=0.0214$

Figure 7 Silencing circ_NOTCH3 diminishes the growth of HCC tumors in vivo. HUH7 cells stably transfected with si-NC or siRNA were transferred into BALB/c nude mice, tumor volume and (A,B) and (C) weight were detected. (D) Tissues were stained by HE solution, magnification 200x. (E,F) The relative expression levels of miR-875-5p and circ_NOTCH3 in xenograft tumors collected from si-NC and siRNA groups were measured by qRT-PCR. (G) Tissues were stained by DAB, magnification 400×; (H) expression of miR-875-5p and circ NOTCH3; (I) expression of miR-875-5p and ZNF146; (J) expression of ZNF146 and circ_NOTCH3. *, P $<0.05$. HCC, hepatocellular carcinoma; HE, hematoxylin and eosin; siRNA, small interfering RNA; qRT-PCR, real-time quantitative reverse transcription polymerase chain reaction; DAB, 3,3'-diaminobenzidine.

\section{Silencing circ_NOTCH3 can diminish the growth of HCC tumors in vivo}

In order to further study whether circ_NOTCH3 silencing can diminish the growth of HCC tumors in vivo, we inoculated HUH7 cells stably transfected with siRNA or si-NC into nude mice. The transplanted tumor was observed 4 weeks after the inoculation. The outcomes revealed that the tumor volume and weight of the siRNA transfection group were evidently lower than those of the matched group (Figure $7 A-7 C$ ). HE staining result showed that downregulation of circ_NOTCH3 inhibited tumor metastasis (Figure 7D). Together, miR-875-5p and circ NOTCH3 were measured in xenograft tumors collected from the siRNA and matched group and miR-875-5p in the siRNA group was evidently up-regulated, while circ NOTCH3 was obviously down-regulated (Figure $7 E, 7 F$ ). Additionally, through immunohistochemical analysis, we discovered that the staining intensity of ZNF146 in the tumor sections of the siRNA-circ_NOTCH3 group was 
evidently weaker than in the matched group (Figure $7 G$ ). Additionally, in xenograft tumors, miR-875-5p was negatively correlated with circ_NOTCH3 or ZNF146 (Figure $7 \mathrm{H}, 7 \mathrm{I}$ ), while circ_NOTCH3 was positively correlated with ZNF146 (Figure 7f). These outcomes indicate that silencing circ_NOTCH3 diminishes the growth of HCC tumors in vivo.

\section{Discussion}

Liver cancer is a highly heterogeneous malignant tumor caused by complex genetic and epigenetic changes. Although the treatment of HCC has been continuously improved in recent years, the overall survival rate of HCC is still low (16). How to improve the prognosis of HCC patients is still the main problem faced in the clinical treatment of HCC. In-depth exploration of the molecular mechanism of the occurrence along with development of HCC and seeking biomarkers for early diagnosis/prognosis of HCC, potential therapeutic targets, and so on, are essential to improve the diagnosis and treatment of HCC. As an emerging ncRNA in recent years, circRNA plays a very important role in the occurrence and development of HCC, for example circMTO1 along with cSMARCA5 can act as competitive endogenous RNA (ceRNA) to bind to cancer-promoting miRNA, inhibit the proliferation and metastasis of HCC, and can be employed as potential therapeutic targets for $\operatorname{HCC}(17,18)$. In this study, we discovered that circ_NOTCH3 is specifically low expressed in HCC specimens. Combined with the clinicopathological parameters of HCC patients and in vitro experiments, we confirmed that circ_NOTCH3 plays an important role in the proliferation of $\mathrm{HCC}$ cells.

As a proto-oncogene, circ_NOTCH3 competes with miR-205-5p, regulates the expression of KLF12, and promotes the development and progression of basallike breast cancer (15); however, its biological function is unclear. In this study, we discovered circ_NOTCH3 is obviously related to the Barcelona Clinic Liver Cancer (BCLC) staging of patients. Based on the BCLC, staging can reflect the tumor status of the patient, and it is widely employed in the preoperative evaluation of HCC patients. We believe circ_NOTCH3 can be employed as potential biomarkers for evaluating tumor burden in patients with HCC. Additionally, our study also found that circ NOTCH3 is obviously down-regulated in HCC tumor specimens, and that it can affect miR-875-5p through the "sponge" effect in HCC cells, thereby indirectly inhibiting cell proliferation. The circRNA/miRNA/mRNA axis is a classic regulatory network for current circRNA functional research, which can regulate gene expression at the transcription and post-transcriptional levels.

MiR-875-5p is a newly discovered key molecule for the malignant transformation of normal cells $(19,20)$. Previous studies involving orthotopic liver cell transplantation models and tumor models induced by high-fat diet have exhibited that the overexpression of miR-875-5p promotes liver cell transformation along with the occurrence of HCC (21). Although interferon- $\beta$ (IFN- $\beta$ ) can act as a regulator of miR-875-5p expression, the direct upstream regulator of miR-875-5p is still unclear (22). Our research showed that circ_NOTCH3 may directly inhibit the activity of tumorpromoting factor miR-875-5p, which in turn affects the proliferation of HCC cells, providing new insights into the upstream regulatory mechanism of miR-875-5p in HCC.

Zinc finger proteins refer to a class of proteins which contain stable $\mathrm{Zn}^{2+}$ and can self-fold to form a "finger"like structure (23). They are the largest family of transcriptional regulatory factors in mammals, and are involved in embryonic development, cell differentiation, cell transformation, and cell cycle (24-26). Studies have discovered most zinc finger proteins are closely related to the occurrence and development of malignant tumors (27). The expression of ZNF23 in the zinc finger protein family in primary HCC is obviously lowered and is closely related to the chemotherapeutic effect of cisplatin. Cisplatin may up-regulate ZNF23 in HCC lines, thereby promoting cell apoptosis (28). Another member of the zinc finger protein family, ZNF146, has been shown to have obviously augmented expression in breast cancer, and as the degree of cell differentiation decreases, its expression augments more obviously, suggesting that ZNF146 has a certain effect on the diagnosis of breast cancer. Le Chalony et al. (29) discovered ZNF146 in liver cancer specimen was obviously augmented by immunohistochemistry, while normal intestinal mucosal epithelium and inflammatory intestinal mucosal epithelium was rarely expressed, suggesting ZNF146 may be closely related to the occurrence of tumors. It is also expressed in the liver, but its relationship with HCC is unclear. The outcomes of this study showed that ZNF146 mRNA and its protein in HCC tumor specimens was obviously higher than that in adjacent specimens, and the difference was statistically significant $(\mathrm{P}<0.05)$, suggesting that $\mathrm{ZNF} 146$ may be involved in the occurrence and evolution of HCC.

These studies revealed circRNA synthesized in vitro 
can inhibit oncogenic miRNAs through its "cavernosal" function, providing new ideas for the development of convenient and effective clinical treatment strategies for tumors. Therefore, we believe the "cavernous body" circ_NOTCH3, which has the function of inhibiting the proliferation of HCC cells discovered in this research, can be employed as a potential therapeutic target for HCC, and its application in the clinical treatment of HCC should be eagerly anticipated.

\section{Acknowledgments}

Funding: None.

\section{Footnote}

Reporting Checklist: The authors have completed the ARRIVE reporting checklist. Available at https://dx.doi. org/10.21037/jgo-21-567

Data Sharing Statement: Available at https://dx.doi. org/10.21037/jgo-21-567

Conflicts of Interest: All authors have completed the ICMJE uniform disclosure form (available at https://dx.doi. org/10.21037/jgo-21-567). The authors have no conflicts of interest to declare.

Ethical Statement: The authors are accountable for all aspects of the work in ensuring that questions related to the accuracy or integrity of any part of the work are appropriately investigated and resolved. Experiments were performed under a project license (No. 2020-011) granted by ethics board of Qingdao No. 6 People's Hospital, in compliance with NIH guidelines for the care and use of animals. All procedures performed in this study involving human participants were in accordance with the Declaration of Helsinki (as revised in 2013). The study was approved by ethics board of Qingdao No. 6 People's Hospital (No. 2020003 ) and informed consent was taken from all patients.

Open Access Statement: This is an Open Access article distributed in accordance with the Creative Commons Attribution-NonCommercial-NoDerivs 4.0 International License (CC BY-NC-ND 4.0), which permits the noncommercial replication and distribution of the article with the strict proviso that no changes or edits are made and the original work is properly cited (including links to both the formal publication through the relevant DOI and the license). See: https://creativecommons.org/licenses/by-nc-nd/4.0/.

\section{References}

1. Casadei-Gardini A, Cascinu S, Colombo M. Atezolizumab and bevacizumab in advanced hepatocellular carcinoma: ready for prime time? Gastroenterology 2021;160:1423-4.

2. Younossi ZM, Corey KE, Lim JK. AGA Clinical practice update on lifestyle modification using diet and exercise to achieve weight loss in the management of nonalcoholic fatty liver disease: expert review. Gastroenterology 2021;160:912-8.

3. Wang X, Fang Y, Yang S, et al. A hybrid network for automatic hepatocellular carcinoma segmentation in H\&E-stained whole slide images. Med Image Anal 2021;68:101914.

4. Malik A, Thanekar U, Amarachintha S, et al. "Complimenting the Complement": mechanistic insights and opportunities for therapeutics in hepatocellular carcinoma. Front Oncol 2021;10:627701.

5. Ren Z, Yue Y, Zhang Y, et al. Changes in the peripheral blood Treg cell proportion in hepatocellular carcinoma patients after transarterial chemoembolization with microparticles. Front Immunol 2021;12:624789.

6. Xiong D, Wang D, Chen Y. Role of the long non-coding RNA LINC00052 in tumors. Oncol Lett 2021;21:316.

7. Liu TJ, Hu S, Qiu ZD, et al. Anti-tumor mechanisms associated with regulation of non-coding RNA by active ingredients of Chinese medicine: a review. Front Oncol 2021;10:634936.

8. Nabih HK. The significance of HCV viral load in the incidence of HCC: a correlation between Mir-122 and CCL2. J Gastrointest Cancer 2020;51:412-7.

9. Deng R, Cui X, Dong Y, et al. Construction of circRNAbased ceRNA network to reveal the role of circRNAs in the progression and prognosis of hepatocellular carcinoma. Front Genet 2021;12:626764.

10. Zhang Z, Han L. Circular RNAs sequenced at last. Nat Biotechnol 2021;39:811-2.

11. Zhang J, Hou L, Zuo Z, et al. Comprehensive profiling of circular RNAs with nanopore sequencing and CIRI-long. Nat Biotechnol 2021;39:836-45.

12. Tijsen AJ, Cócera Ortega L, Reckman YJ, et al. Titin circular RNAs create a back-splice motif essential for SRSF10 splicing. Circulation 2021;143:1502-12.

13. Circular RNA. Nat Biotechnol 2021;39:23.

14. Luo Y, Fu Y, Huang R, et al. CircRNA_101505 sensitizes 
hepatocellular carcinoma cells to cisplatin by sponging miR-103 and promotes oxidored-nitro domain-containing protein 1 expression. Cell Death Discov 2019;5:121.

15. Guan B, Li Q, Zhang HZ, et al. circ_NOTCH3 functions as a protooncogene competing with miR205-5p, modulating KLF12 expression and promoting the development and progression of basal-like breast carcinoma. Front Oncol 2021;10:602694.

16. Wei L, Owen D, Rosen B, et al. A deep survival interpretable radiomics model of hepatocellular carcinoma patients. Phys Med 2021;82:295-305.

17. Han D, Li J, Wang H, et al. Circular RNA circMTO1 acts as the sponge of microRNA-9 to suppress hepatocellular carcinoma progression. Hepatology 2017;66:1151-64.

18. Yu J, Xu QG, Wang ZG, et al. Circular RNA cSMARCA5 inhibits growth and metastasis in hepatocellular carcinoma. J Hepatol 2018;68:1214-27.

19. Kang N, Ou Y, Wang G, et al. miR-875-5p exerts tumorpromoting function via down-regulation of CAPZA1 in esophageal squamous cell carcinoma. PeerJ 2021;9:e10020.

20. Ye L, Yu Y, Zhao Y. Icariin-induced miR-875-5p attenuates epithelial-mesenchymal transition by targeting hedgehog signaling in liver fibrosis. J Gastroenterol Hepatol 2020;35:482-91.

21. Xu Q, Zhu Q, Zhou Z, et al. MicroRNA-876-5p inhibits epithelial-mesenchymal transition and metastasis of hepatocellular carcinoma by targeting BCL6 corepressor like 1. Biomed Pharmacother 2018;103:645-52.

22. Zhang T, Cai X, Li Q, et al. Hsa-miR-875-5p exerts tumor suppressor function through down-regulation of EGFR in colorectal carcinoma (CRC). Oncotarget 2016;7:42225-40.

23. Zhang Y, Xia H, Yi W, et al. Experimental study on the effect of zinc finger protein A20 on lumbar intervertebral disc degeneration in rabbits. Zhongguo Xiu Fu Chong Jian Wai Ke Za Zhi 2021;35:366-74.

24. Lv Z, Qi L, Hu X, et al. Zic family member 2 (ZIC2): a potential diagnostic and prognostic biomarker for pancancer. Front Mol Biosci 2021;8:631067.

25. Yan N, Liu C, Tian F, et al. Downregulated mRNA expression of ZNF385B is an independent predictor of breast cancer. Int J Genomics 2021;2021:4301802.

26. Ichida $Y$, Utsunomiya $Y$, Onodera $M$. Effect of the linkers between the zinc fingers in zinc finger protein 809 on gene silencing and nuclear localization. Biochem Biophys Res Commun 2016;471:533-8.

27. Schuster A, Klein E, Neirinckx V, et al. AN1-type zinc finger protein 3 (ZFAND3) is a transcriptional regulator that drives Glioblastoma invasion. Nat Commun 2020;11:6366.

28. Shi Y, Zheng L, Luo G, et al. Expression of zinc finger 23 gene in human hepatocellular carcinoma. Anticancer Res 2011;31:3595-9.

29. Le Chalony C, Prospéri MT, Haluza R, et al. The OZF gene encodes a protein consisting essentially of zinc finger motifs. J Mol Biol 1994;236:399-404.

(English Language Editor: J. Jones)
Cite this article as: Bao L, Wang M, Fan Q. Hsa_circ_ NOTCH3 regulates ZNF146 through sponge adsorption of miR-875-5p to promote tumorigenesis of hepatocellular carcinoma. J Gastrointest Oncol 2021;12(5):2388-2402. doi: 10.21037/jgo-21-567 